\title{
Self-Organising Assembly Systems formally specified in Maude
}

\author{
Regina Frei • \\ Traian Florin Șerbănuță . \\ Giovanna Di Marzo Serugendo
}

Received: date / Accepted: date

\begin{abstract}
This article recapitulates on the research done in Self-Organising Assembly Systems (SOAS) and present the completed formal specifications and their simulation in Maude. SOAS are assembly systems that (1) participate in their own design by spontaneously organising themselves in the shop floor layout in response to the arrival of a product order and (2) manage themselves during production. The self-organising process for SOAS to design themselves follows the Chemical Abstract Machine (CHAM) paradigm: industrial robots self-select and self-arrange according to specific chemical rules in response to a product order with generic assembly instructions (GAP). This article presents an additional set of rules describing how the GAP is transformed into Layout-Specific Assembly Instructions (LSAI) which is a kind of recipe for how the self-organising robots assemble the product.
\end{abstract}

\section{R. Frei}

EPSRC Centre for Innovative Manufacturing

in Through-life Engineering Services

Cranfield University

Bedfordshire MK43 0AL, United Kingdom

E-mail: work@reginafrei.ch

T.F. Serbănută

Formal Systems Laboratory

Department of Computer Science

University of Illinois at Urbana-Champaign

2111A Siebel Center, 201 N. Goodwin

Urbana, IL, USA, 61801

E-mail: tserban2@illinois.edu

G. Di Marzo Serugendo

Institute of Services Science

Faculty of Social and Economics Science

University of Geneva

1227 Carouge, Switzerland

E-mail: giovanna.dimarzo@unige.ch
Keywords Self-organisation · Maude · Agile manufacturing · Formal specifications · Assembly systems

\section{Introduction}

Self-Organising Assembly Systems (SOAS) represent an approach which gives agile manufacturing systems more intrinsic intelligence to serve the user in a more autonomous way. This is a very valuable approach in a time where technological systems are becoming increasingly complex, difficult to manage and laborious to change. In response, a development towards systems with more autonomy can be observed in many different areas of engineering and technology. This includes Autonomic Computing (Kephart and Chess 2003), Complexity Engineering (Frei and Di Marzo Serugendo 2011b,a, 2012), Emergent Engineering (Ulieru and Doursat 2011), many types of self-healing technologies (Frei et al. 2012), selfassembly at various scales (Boncheva et al. 2003; Gross and Dorigo 2008; Phili and Stoddart 1996) and many other self-* approaches.

Additionally, manufacturing needs to become more agile and responsive to change in a highly competitive world. Production lot sizes tend towards very small numbers, whereas the product variants and options are getting more diverse. Manufacturing and assembly systems therefore need to be agile, highly responsive to changing requirements, and able to function with minimal downtime for reconfiguration and maintenance. These objectives are addressed in the paradigm of Evolvable Assembly Systems (EAS) (Barata 2005; Onori 2002; Onori et al. 2008, 2011). SOAS (Frei 2010; Frei and Di Marzo Serugendo 2011c) go a step further by assigning the system modules a proactive role in system design and assembly execution. 
This article presents the now completed work done based on what was previously published: This includes the design (Frei et al. 2008a, 2010b) (Di Marzo Serugendo and Frei 2010) and the architecture (Frei et al. 2009) of SOAS (Frei 2010) (Frei and Di Marzo Serugendo 2011c), as well as the development of a specific ontology and 'on-the-fly' creation of coalitions (Frei et al. 2008b). A brief summary is in (Di Marzo Serugendo and Frei 2012). In (Frei et al. 2010a) we explained the various relevant concepts, which are briefly reviewed in this article, clarified the relation between Ambient Intelligence and SOAS, and presented the first part of the formal specifications. This work has now been completed and serves as a proof of concept.

SOAS and Ambient Intelligence: Ambient intelligence (ISTAG 2001, 2003) refers to electronic systems that are sensitive and responsive to the presence of people. They are usually embedded in the everyday environment, context-aware, personalised, adaptive or anticipatory to changes in the environment.

In the area of manufacturing and assembly, a product designer traditionally provides a design of the product, this is an assembly sequence technically describing how to join the products parts and in which order. A team of engineers then builds an appropriate assembly system, which consists of a series of modules or complete robots, properly arranged and connected in the shop-floor layout, and programmed to execute the assembly movements.

Traditionally, the resulting assembly system is dedicated to building the specified product and runs under central control. A human operator monitors the system, its performance and the quality of products. The system is equipped with security features that stop the system in case of critical failures. The operator must then find out what went wrong and how to fix it.

Evolvable and self-organising assembly systems revisit this way of building systems and demonstrate Ambient Intelligence features and responsiveness to people along the following three lines (Frei et al. 2010a):

- Product requirements and link with the product designer. A new assembly sequence, provided by the product designer, triggers a self-organising process among the different modules available either from the storage or already positioned on the shop-floor. The modules spontaneously organise into appropriate coalitions (groups of modules) to fulfill the tasks specified in the assembly sequence.

The assembly processes and the assembly system technology can even iteratively influence the product design (Onori 2002). The product designer and the assembly system collaborate in producing the final product design.

- Layout design and link with the engineer building the assembly system. The modules autonomously search for suitable coalition partners to compose the skills required to fulfill the tasks of the assembly sequence. Additionally, the coalitions arrange themselves in the shop-floor layout: they choose a position taking into account overlapping workspaces. The engineer may also collaborate to this process and suggest some specific module or preferred position. The obtained layout and final choice of modules is validated by the engineer. The assembly system resulting from the collaboration between the engineer and the self-organising modules is then able to execute the assembly sequence.

- Production and link with the operator. During production, the assembly system performs monitoring tasks. The individual modules participating in the assembly system monitor themselves, their neighbours, the quality of the produced products, any unexpected item (human hand, dropped part). Some of these tasks involve high precision, mini and/or micro-movements, checking of performances that go beyond human capabilities. In collaboration with the operator, who can stop/reset the system at any time, the assembly system runs the production at the best possible performance given the current production conditions.

The technology involved encompasses: RFID tags attached to individual products being assembled reporting on assembly tasks performed so far, sensors attached to the different modules reporting on performances such as precision or speed, and autonomous software agents acting as wrappers around the physical modules enabling them to become reactive and adaptive.

Organisation of this article: Section 2 briefly introduces rewriting logic, the Chemical Abstract Machine and the Maude software. Section 3 details the case study used in this paper. Section 4 recalls the previously published specifications and indicates differences between the previous and the new work. Section 5 presents the completed specification and simulation results. Finally, Section 6 concludes this article.

\section{Background and previous work}

This section is intentionally kept short. For more details, please consult (Frei et al. 2010a).

The Gamma chemical reaction model (Banâtre et al. 2000) was introduced as an alternative to sequential models of programs. Gamma is built around the 
idea of a chemical reaction metaphor. The main data structure is the multiset seen as a chemical solution. A program is then a pair (ReactionCondition, Action). The execution consists of removing from the set the elements that appear in the ReactionCondition part and replacing them with the product of the Action. The program stops and reaches a stable state when no more reactions can take place.

The Chemical Abstract Machine (CHAM) (Berry and Boudol 1998) is an extension of the Gamma model allowing modular structures: chemical reactions may occur within membranes and stay local to the membrane; and the opposite operation, the airlock, allows the extraction of a molecule from a membrane.

Rewriting logic was introduced by Meseguer (Meseguer 1990, 1992) as a fundamental logic for concurrency, modelling transitions between equationally defined congruence classes of terms. Rewriting logic extends equational logic with rewrite rules, allowing one to derive both equations and rewrites (or transitions). Deduction remains the same for equations (i.e., replacing equals by equals), but the symmetry rule is dropped for rewrite rules. Formally, a rewrite theory is a triple $(\Sigma, E, R)$, where $(\Sigma, E)$ is an equational specification and $R$ is a set of rewrite rules. Rewriting logic is a framework for true concurrency: the locality of rules, given by their context-insensitiveness, allows multiple rules to apply at the same time provided their patterns don't overlap.

Maude (Clavel et al. 2007) is a rewrite engine offering full execution and analysis support for rewriting logic specifications ${ }^{1}$. Maude provides an execution and debugging platform, a breadth-first search (BFS) statespace exploration, and a linear temporal logic (LTL) model checker (Eker et al. 2003), as well as an inductive theorem prover (Clavel et al. 2006) for rewrite logic theories; these translate immediately into corresponding BFS reachability analysis, LTL model checking tools, and theorem provers for the defined models. For example, these generic tools were used to derive a competitive model checker (Farzan et al. 2004), and a Hoare logic verification tool (Sasse and Meseguer 2007) for the Java programming language.

CHAM within rewriting logic: The Chemical Abstract Machine can be viewed as a particular definitional style within rewriting logic (Meseguer 1992; Șerbănuță et al. 2009). That is, every CHAM is a specific rewrite theory in rewriting logic, and CHAM computation is precisely concurrent rewriting computation.

1 This work was implemented in Maude version 2.4.

\section{Case study example of SOAS}

For illustration purposes, we assume the following simple product to be assembled: an adhesive tape roller dispenser, consisting of two body case parts part $_{1}$ and part $\left._{3}\right)$, a tape roll $\left(\right.$ part $\left._{2}\right)$ and a screw $\left(\right.$ part $\left._{4}\right)$, assembled on top of a carrier, as shown in Figure 1. For more details see (Frei 2010).

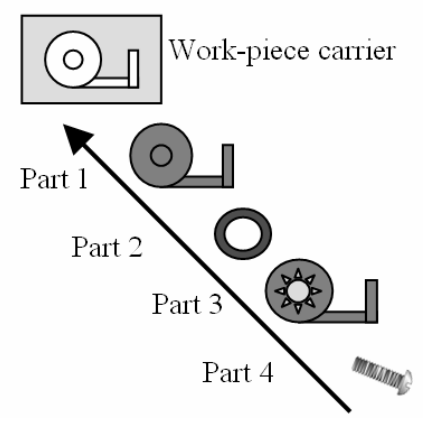

Fig. 1 The adhesive tape roller dispenser

The Generic Assembly Plan (GAP) specifies the way a product is to be assembled: it includes the assembly sequence of the different parts and the way they must be joined. Tasks are defined in the form of generic operations (equivalent to skills). The GAP does not provide information about what module ${ }^{2}$ to use and what movement to make. In other words, the GAP says what to do (assemble 10 tape rollers by joining part $_{1}$ with part $_{2}$, part $_{3}$ and part $_{4}$ ) but not how (which robotic modules handle which parts and execute which movements at which instant) and is thus independent from any layout. Figure 2 shows the example of a GAP represented as a workflow and written in XML.

The six simple tasks illustrated in Figure 2 each have an operation type (Op), an object to be handled (Obj), a start point (StPt), an end point (EndPt), as well as a start orientation (StOr) and an end orientation (EndOr), referring to the parts to be treated. We assume this to be sufficient information at this stage of implementation. This GAP specifies that a carrier is loaded from the storage to conveyor, then part $_{1}$ is picked from feeder $_{1}$ and placed on the carrier, then part $_{2}$ is picked from feeder $_{2}$ and placed on top of part $_{1}$. The same procedure follows for part $_{3}$ that is placed on top of part $_{2}$, and part $_{4}$ that is screwed into part 3 . Finally, the carrier with the assembled product is unloaded to the storage. The flash in the rectangle on the left hand side of the GAP represents the beginning $(I N)$, and

2 with the exception of the feeders, which are part-specific 


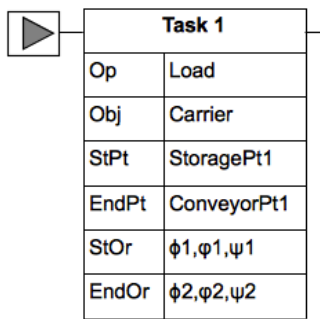

\begin{tabular}{|l|l|}
\hline \multicolumn{2}{|c|}{ Task 2 } \\
\hline Op & Pick\&Place \\
\hline Obj & Part1 \\
\hline StPt & Feeder1Pt \\
\hline EndPt & CarrierPt \\
\hline StOr & $\phi 3, \varphi 3, \Psi 3$ \\
\hline EndOr & $\phi 4, \varphi 4, \Psi 4$ \\
\hline
\end{tabular}

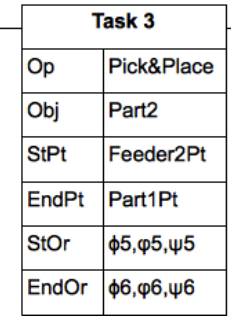

\begin{tabular}{|l|l|}
\hline \multicolumn{2}{|c|}{ Task 4 } \\
\hline Op & Pick\&Place \\
\hline Obj & Part3 \\
\hline StPt & Feeder3Pt \\
\hline EndPt & Part2Pt \\
\hline StOr & $\phi 7, \varphi 7, \Psi 7$ \\
\hline EndOr & $\phi 8, \varphi 8, \Psi 8$ \\
\hline
\end{tabular}

\begin{tabular}{|l|l|}
\hline \multicolumn{2}{|c|}{ Task 5 } \\
\hline Op & Pick\&Place \\
\hline Obj & Part4 \\
\hline StPt & Feeder4Pt \\
\hline EndPt & Part3Pt \\
\hline StOr & $\phi 9, \varphi 9, \psi 9$ \\
\hline EndOr & $\phi 10, \varphi 10, \psi 10$ \\
\hline
\end{tabular}

\begin{tabular}{|l|l|}
\hline \multicolumn{2}{|c|}{ Task 6 } \\
\hline Op & Unload \\
\hline Obj & Product, carrier \\
\hline StPt & ConveyorPt2 \\
\hline EndPt & StoragePt2 \\
\hline StOr & $\phi 11, \varphi 11, \psi 11$ \\
\hline EndOr & $\phi 12, \varphi 12, \psi 12$ \\
\hline
\end{tabular}

Fig. 2 Example of a GAP written as a workflow

the square on the right hand side stands for the end (OUT).

The layout is incrementally built according to a self-organising process illustrated in Figure 3: modules self-assemble to form coalitions according to a process of reactions and rewriting. Coalitions are built to progressively match with the tasks defined in the GAP.

The details of the modelling of this self-organising process leading to such a layout can be found in (Frei et al. 2010a); a summary is here above in Section 2.

To assemble the product, the GAP needs to be transformed into Layout-Specific Assembly Instructions (LSAI) as shown in Figure 4. This transformation takes into account the actual modules, the tasks and the parts. The LSAI consists of executable programs for each of the robotic modules in the coalitions, based on their requested skills. The instructions are generated for a certain layout; if the layout is modified, these instructions must be changed.

At production time, the assembly of a product will result from the execution of the LSAI by the agents / modules according to the workflow. Any change requiring a layout reconfiguration restarts the self-organising layout design process.

Figure 6 illustrates the assembly procedure on a hypothetical linear layout.

\section{Previously published specifications}

The specifications recalled in this section were published in (Frei et al. 2010a) and are necessary for understanding the new work published in Section 5. The first four of the following six rules (Frei and Di Marzo Serugendo 2011c; Frei 2010) have been specified previously; the last two are specified in this article.

1. Interface compatibility

2. Composition patterns

3. Creation of composite skills

4. Task coalition matching

5. Layout design and transport linking

\section{Transforming the GAP into the LSAI (rewriting)}

Note that Maude works with so-called modules, written as $\bmod (\ldots)$ endm. This is an unfortunate coincidence with the typical use of the word module in this article, which refers to a manufacturing resource agent (MRA).

Similarly, note that the term agent used in this context is not to be confused with software agents mentioned elsewhere.

The entire specification will not be discussed in detail here; it can be downloaded from:

http://code.google.com/p/soas-maude.

\subsection{Structure of the model}

The language of our model consists of nested "cells", which are named CHAM-like molecules. Cells contain either concrete values, like Integers, Floats, Names, and enumerations, or a "soup" of other cells, representing CHAM-like configuration molecules.

The top configuration cell, shown in Figure 5, contains three mandatory cells:

- gaps, specifying the General Assembly Plans

- mras, specifying the Manufacturing Resource Agents

- and parts, specifying the Parts

- as well as an optional cell, which appears during the model simulation, which contains the agents, (partial) coalitions of modules aiming to solve a certain task.

For details about other items, please refer to (Frei et al. 2010a).

4.2 Differences from the previously published specifications

The original specifications have been refined and improved; they are now richer, more detailed, and more realistic. 


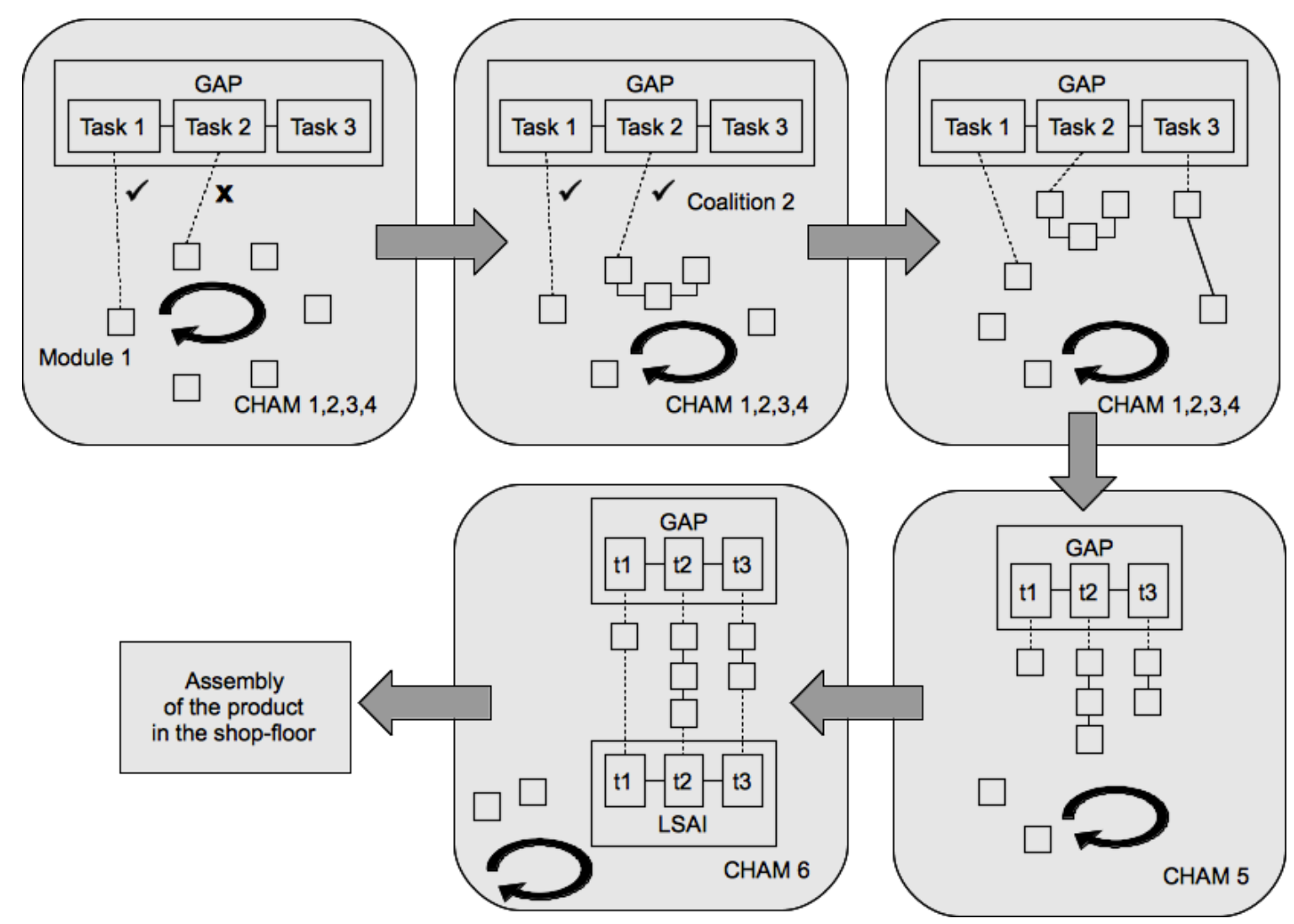

Fig. 3 Self-assembly of coalitions in CHAM, illustrating the process phases dominated by rules 1 to 4 as well as 5 and 6 .

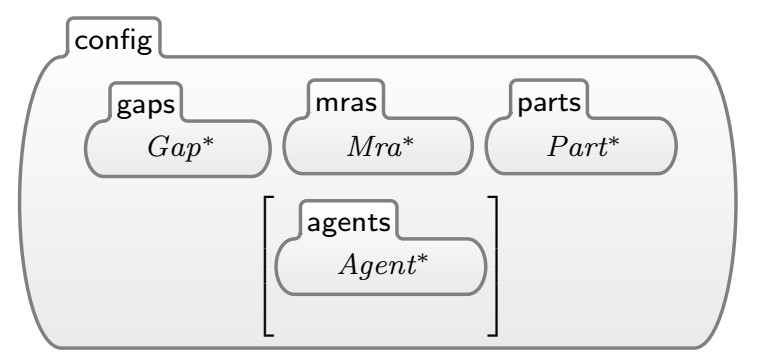

Fig. 5 The top configuration cell

In the previous version (Frei et al. 2010a), the case study only included two parts, namely a body case and the adhesive tape roller. In this extended version, it consists of four parts, with an additional body case part and a screw which holds them together. Moreover, in the previous version, only four of the six types of rules to determine the process of self-organisation were formally specified, whereas now, all six are included. The current version is complete and allows the assembly system to fully design itself from the arrival of a product order (under the form of a GAP) to a complete assembly system ready to work on the pieces and assemble products.
The specifications now include simple rules for layout formation, which guide the robots towards arranging themselves in a serpentine form, with the feeder always being on the left of the robot. This was an initial choice we took for experimenting the concept, and only serves as an example at this stage. Any number of other configuration rules could be specified, such as, for instance, "if the base axis is pneumatically actuated, the feeder places itself on the right of the robot base, with a distance of $10 \mathrm{~cm}$ ".

As a perspective for practical use of these specifications, a template for different layout creation rules / LSAI derivation rules could be provided, so that the user could give their wishes as input. The system would then be guided to design itself according to the requirements of specific production facilities and orders. There could be many different patterns for combining feeders, robots and robotic modules, grippers and conveyors, and they could arrange themselves in any specified shop-floor layout.

The previously published version of our work specified the following:

- MRA (manufacturing resource agent)

- Skills

- Positions, angles 


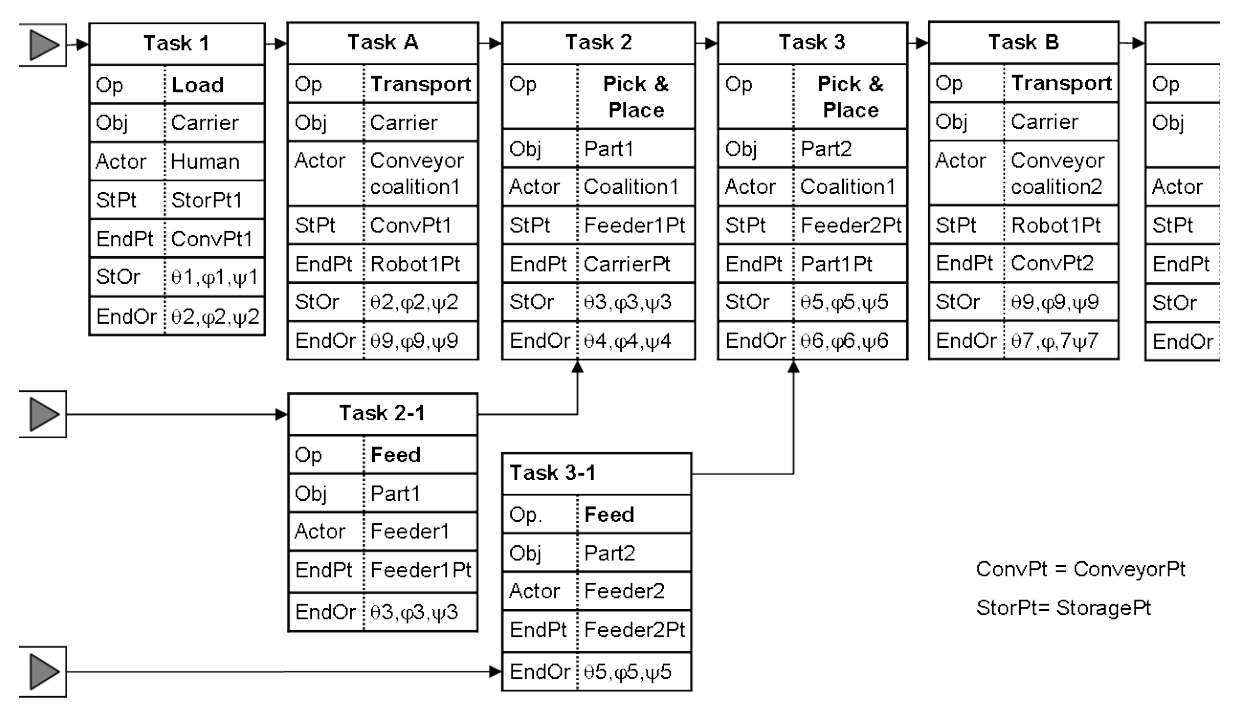

Fig. 4 Example of an LSAI written as a workflow (incomplete view)

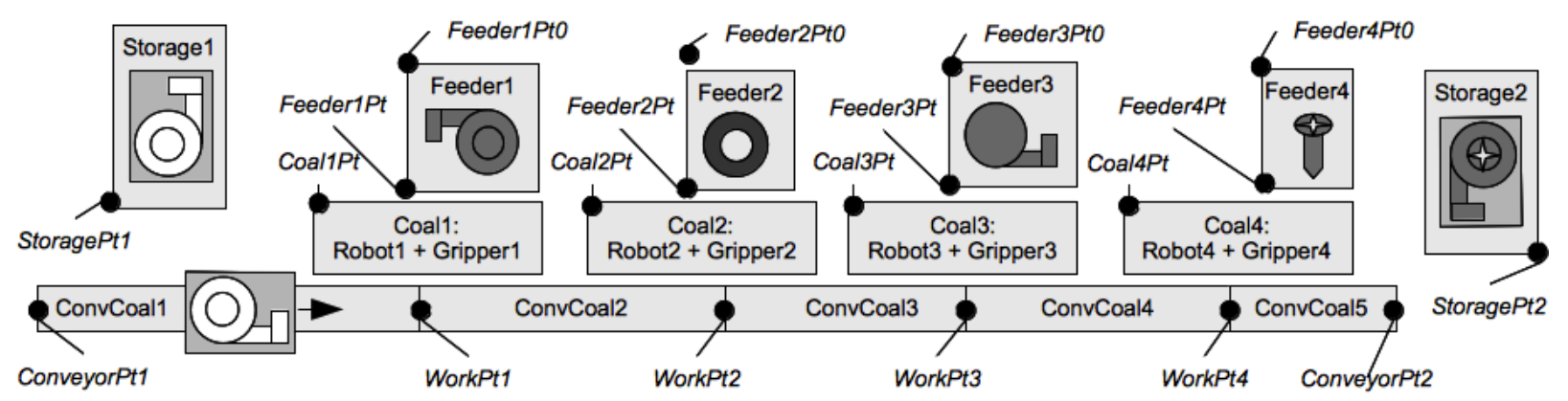

Fig. 6 Concept for a hypothetical linear assembly line

- Task

- GAP (generic assembly plan)

- LSAI (layout-specific assembly instructions)

- Interfaces (+/-) and diverse types

- Parts to be assembled

- Reaction rules:

1) Interface compatibility: describing the physical compatibility of the modules.

2) Typical composition patterns: certain modules are typically combined with certain other modules, e.g. a gripper is always held by a robotic axis (either an individual robotic module or a complete robot). 3) Creation of composite skills: the modules which form a coalition contribute their individual skills and form composite skills, e.g. a gripper can open and close, an axis can move, and together they form a composite pick\&place skill.

4) Task - coalition matching (considering only the type of skills required): a task which requires a certain skill will associate with a coalition that offers those skills.
New features in the additional rules detailed in this article:

Additional reaction rule: 5) Layout creation and transport linking: the chosen modules and coalitions are arranged to form a shop floor layout and connected through conveyors (or potentially other means of transportation).

- Form the layout (robots and feeders choose positions in the layout).

- Establish their geographical arrangement; link robots with conveyors.

- If impossible, adjust robot positions.

- IN and OUT to mark the inputs and outputs of a layout.

- Limit the search space by taking into account user preferences or other constraints.

- Adapt / reconfigure the layout in case of problems; how to determine a solution with minimal changing effort? 
Additional reaction rule: 6) Derivation of the LSAI from the GAP: the concrete assembly movements need to be made explicit for the chosen layout.

Interface verification: Verifying that the interfaces which are about to connect have opposite polarities (either '+/-' or ' $-/+$ ') $\rightarrow$ extending rule 1 )

Gripper type: Matching the gripper-type, subtype and range with the material and size of the part to be moved $\rightarrow$ extending rule 4 )

Indirect requirements: Taking into account the requirements of the modules (for instance, a middle axis will need a base axis) $\rightarrow$ extending rule 2 ) and 3 )

Coalition selection: Rules for deciding which coalition will be associated with a task, if there are more than one possibilities (till now, the first solution was taken) $\rightarrow$ extending rule 4 )

The CHAM-based part of the process ends once suitable modules have associated with tasks to be fulfilled. The layout creation and then the LSAI derivation are based on simple rules, which are being defined using functional Maude equations.

\section{Completed specification and simulation results}

\subsection{Available modules}

The following modules were specified and are thus available in the hypothetical shopfloor repository to build a layout:

- 3 industrial robots abb-robot, cartesian-robot, and scara-robot;

- Base axes $a_{1}, a_{2}, a_{3}$ (having only one interface, the other connection being the ground), middle axes $a_{4}$, $a_{5}, a_{6}$, and top-axes $a_{7}, a_{8}, a_{9}$ (able to hold a gripper);

- Conveyors (generic);

- Endings of the layout ending-in and ending-out;

- Feeders $f_{1}, f_{2}, f_{3}$ and $f_{4}$;

- Grippers $g_{1}, g_{2}, g_{3}, g_{4}$, and $g_{5}$;

- Positioning devices $p d_{1}, p d_{2}, p d_{3}$ and $p d_{4}$ (allowing the conveyors to place the carrier correctly under the robots);

- Humans (generic); technically, the human is wrapped by an MRA with the skills load and unload.

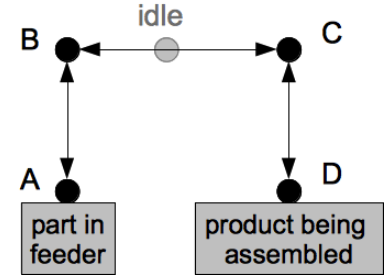

Fig. 7 Positions A, B, C, D, and idle

These modules were chosen because they are suitable to execute the tasks specified in the GAP. Like the product chosen for this case study, the modules only represent an example; any other set of modules could be specified according to the user's needs and the existing equipment. Not all available modules are necessarily used in the layout. For efficiency reasons, humans and conveyors were abstracted; there are as many of them available as required.

\subsection{Pick\&place operation}

Figure 8 illustrates the possible trajectories of a robot executing a pick\&place operation. The movement sequence is normally as follows, with the positions illustrated in Figure 7:

- Preparatory phase : From idle position or any other position, move to B. If gripper closed: open gripper.

- Phase 1: From B move to A, close gripper, move to back to B.

- Phase 2: From B move to C, or the other way round.

- Phase 3: From C move to B, open gripper, move to back to $\mathrm{C}$. If no other task to execute: move to idle position and close gripper.

Furthermore, Figure 8 indicates the robotic skills which could execute the required movements. For Phase 2 , for instance a robot capable of moving linearly in the horizontal plane (lin $\mathrm{x}$, lin $\mathrm{y}$ ) would be suitable. Alternatively, also a robotic module with the vertical $\mathrm{z}$ axis as a rotational axis may be used. For reasons of simplicity, different solutions with other modules or other module combinations are not considered here. For Phases 1 and 3, an axis that moves linearly along the $\mathrm{z}$ axis or one that rotates along a horizontal axis (no matter if $\mathrm{x}$ or y) may be used. Again, this choice is not exhaustive and motivated by the intention to simplify the model.

In this sense, the generation of composite skill 'pick\&place' is currently hardcoded in the rules. This is suitable because the combination of gripper and axes follows a typical composition pattern. For less common module combinations - for instance, combining a full industrial 


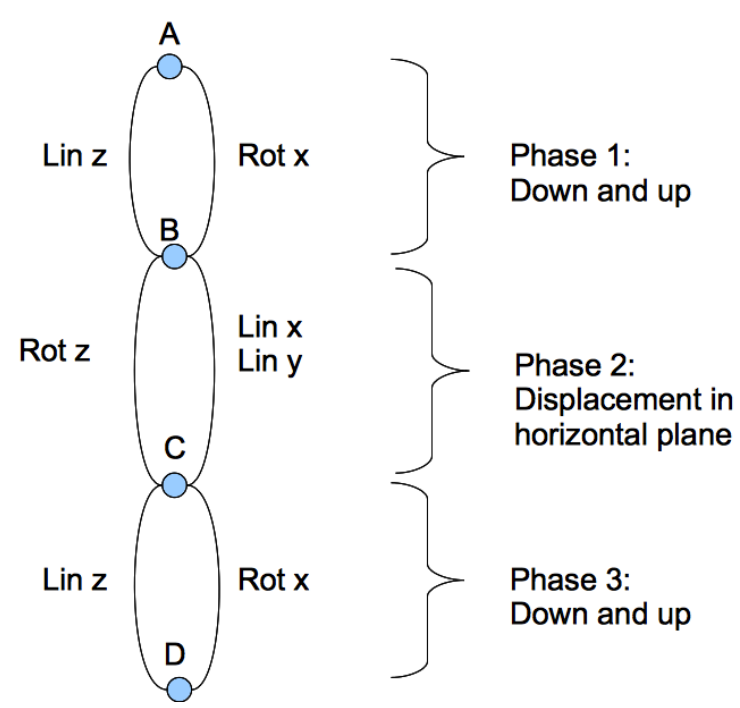

Fig. 8 Robot trajectories in a 'pick\&place' operation and suitable movements; abstract representation showing the combinatoric possibilities to combine robot movements.

robot with an additional axis, other rules could be introduced; there is even some space for the emergence of new opportunistic combinations.

The Maude rules defining the LSAI generation for the pick\&place operation are presented in Section 5.4

\subsection{Rules for layout design and transport linking}

Figure 9 illustrates the result of the specification execution, based on a set of simple rules, as follows:

- Start at $(0,0,0)+$ offset, going eastwards (rule in Figure 10)

- Add a coalition towards the direction of advancement if there is enough space (e.g., rule in Figure 12)

- If end of floor is reached, add a conveyor corner, then a conveyor going north, another conveyor corner, and change the direction (e.g., rule in Figure 13)

- Stop when all coalitions have been placed

Any other strategy or rules for building a layout could be specified according to the user's preferences and the actual shop floor constraints.

In our current Maude implementation, the layout derivation begins at the end of the process of assigning tasks to the formed MRA coalitions. Once a set of agents covering all the tasks was successfully chosen, the system transitions into the layout state. Once this happens, the rule in Figure 10 is triggered, setting the environment for the layout and LSAI generation phases. This rule sets up a nested structure of cells which will trigger the layout and LSAI specific rules. For example, the layout generation cell is initialized with the agents resulted after the coalition formation phase, as well as with cells containing necessary metadata information such as the beginning position set to the south-west corner or the direction of advancing set to east. These cells are conceived to dissolve once all the agents have been processed in that stage; for example, Figure 11 presents the rule concluding the layout generation phase.

A typical rule for setting the position of an agent is presented in Figure 12. The rule first checks in the side condition whether there is enough room on the shopfloor in the direction of advancing (here east). If so, it creates a new agent to represent the conveyor taking the product from its previous station to the current one and then it sets the position of the current agent at the end of the newly added conveyor. At the same time, the current position is updated to the right of the positioned agent. Note that in this rule each agent and carrier gets assigned an id identifying its position in the assembly line. The rules for the west direction are similar.

When the end of the shop floor is reached (either towards west or east), a corner rule applies. Figure 13 presents the rule for changing direction of the assembly line when the west end of the shop floor is reached. The rule first checks in the side condition if adding a new agent in the same direction will exceed the limits of the shop floor (here westwards), and if this is the case, it verifies that the shop floor has enough space to continue northwards. If so, three new conveyors are positioned: a corner one, to take the product from the previous conveyor and orient it northwards, then a regular conveyor to carry the product north, followed by another corner conveyor to change the direction to the opposite of the original one (here from west to east).

\subsection{Rules for tranforming the GAP into the LSAI}

Based on the GAP and the created layout, a corresponding LSAI needs to be generated. This is done according to a set of rules which can be modified depending on user preferences and robot characteristics.

Taking into consideration the coordinates of each module's position in the layout, the previously abstract movements are now instantiated. Abstract points become concrete, and skills become operations.

For example, the main rule for deriving the pick\&place movement sequence described in Section 5.2 is formalized in our Maude implementation by the following equation:

eq createP\&PMovements(〈agent $\rangle$ AgentIs $\langle/$ agent $\rangle$, AP, DP, Skills)

$=\langle$ agent $\rangle$ AgentIs $\langle$ procedure 


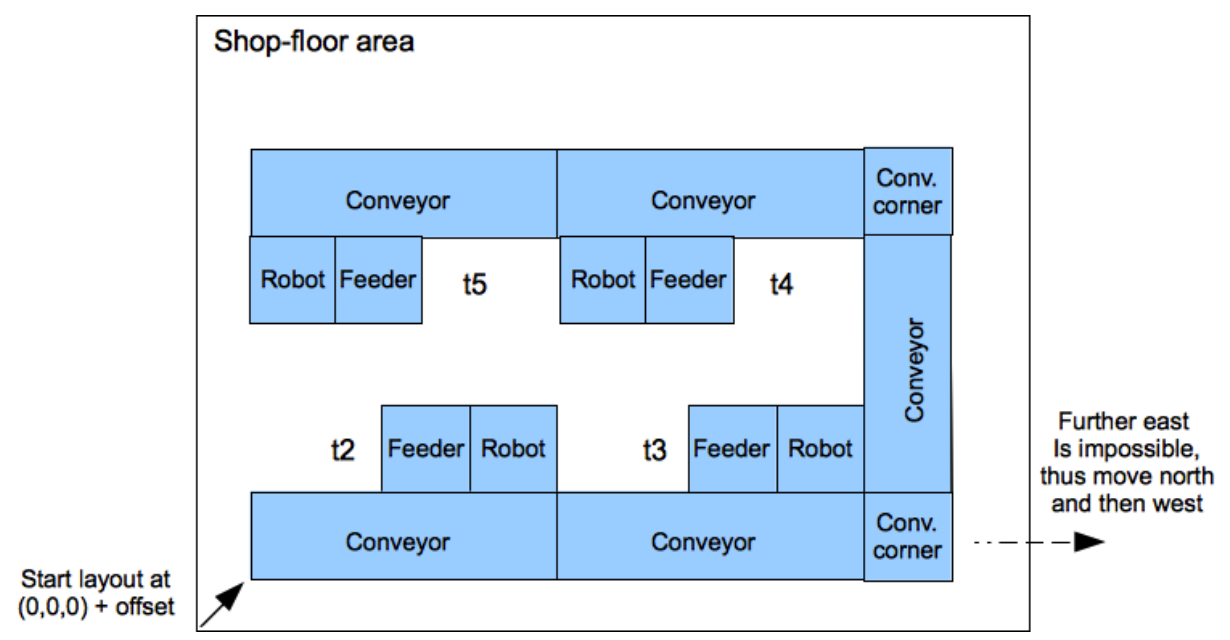

Fig. 9 Layout generated with a set of simple rules inspired by chemical abstract machine, representing an example of a more general case. The first task t1 (loading) and the last task t6 (unloading) are executed by humans and therefore not shown. To maintain the generic nature of the illustration, the robots and grippers are not given specific identifications. The form of the layout was determined by layout formation rules that were chosen for no particular reason; they could be easily replaced by any other layout formation rules and are classified as 'user preferences'.

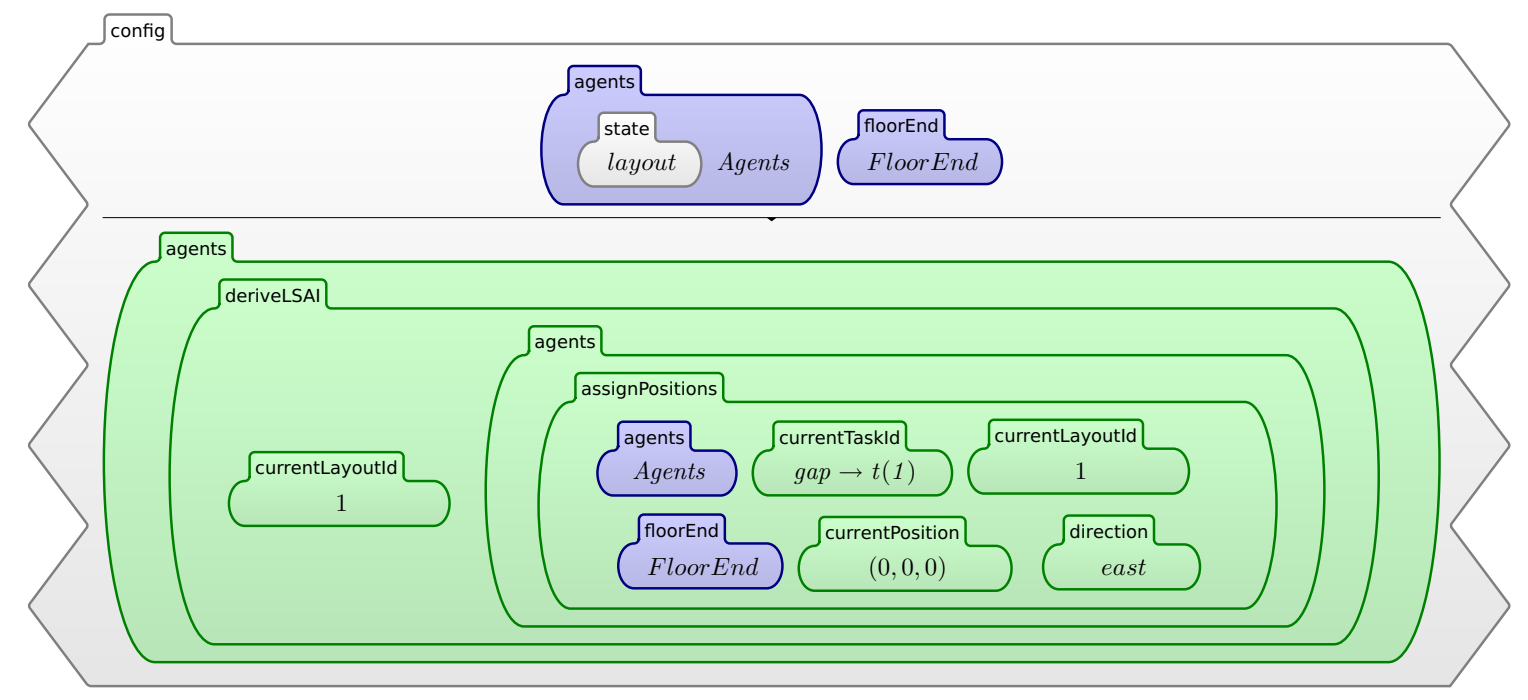

Fig. 10 Rule for starting the layout and LSAI plan generation

moveDown(AP,Skills),

$\langle$ skill $\rangle$

$\langle$ type $\rangle$ open-close $\langle/$ type $\rangle\langle$ subtype $\rangle$ close $\langle/$ subtype $\rangle$ $\langle/$ skill $\rangle$

moveUp(AP, Skills),

moveFromTo(AP, DP, Skills, -1.0),

moveDown(DP, Skills),

$\langle$ skill $\rangle$

$\langle$ type $\rangle$ open-close $\langle/$ type $\rangle\langle$ subtype $\rangle$ open $\langle/$ subtype $\rangle$ $\langle/$ skill $\rangle$,

moveUp(DP, Skills),

moveFromTo(DP, AP, Skills, 1.0)

$\langle/$ procedure $\rangle$

$\langle/$ agent $\rangle$.
This equation gives a high-level formalisation of the pick and place procedure, by translating it in concrete movements and open/closing of grippers executed by the MRAs coalition with its available skills. The idle position is $\delta_{z}$ above the middle of the feeder with the gripper open. From this position the coalition uses the available Skills to moveDown to the middle of the feeder (position AP), then it uses the open-close skill of the gripper to close and grip the part being fed, then it first moves back up to the idle position (moveUp), then in the horizontal plane (moveFromTo) above the position of the conveyor (position DP), where it descends (moveDown) and it opens the gripper to place the part 


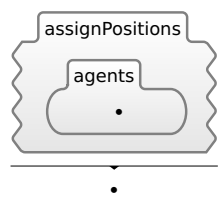

Fig. 11 Rule for concluding the layout generation phase.
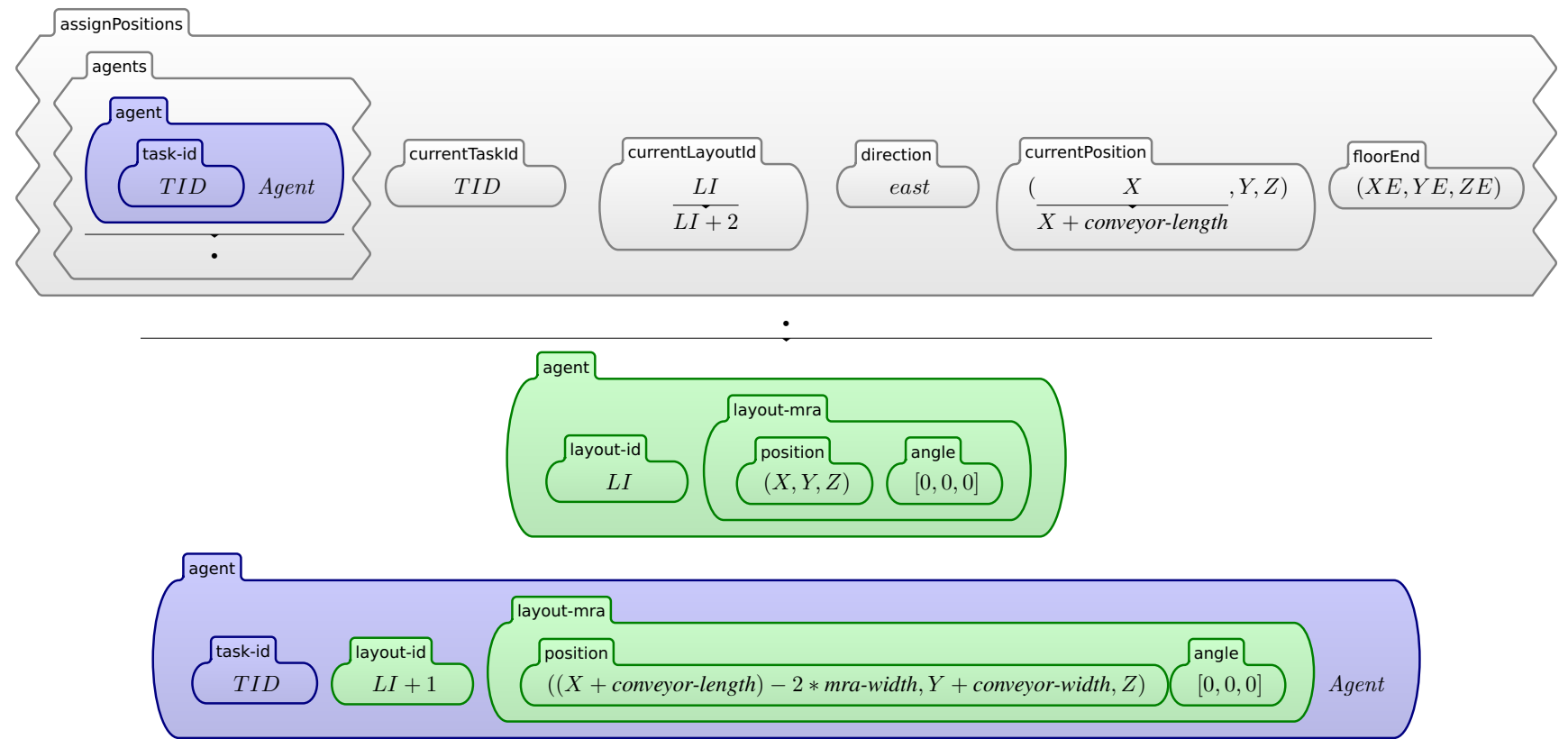

when $X+$ conveyor-length + conveyor-width $\leq X E$

Fig. 12 Setting an agent in the layout

on top of the current product. Finally, it retraces its moves up and then back to the idle position. Figure 7 illustrates this sequence of movements.

As mentioned above, the precise movements are computed based on the available skills, and could be a composition of linear and rotational movements, with preference given to linear movements. For example, the equational specification for the moveDown operation consists of the two conditional equations depicted below, the first used when linear movement skills are available, while the second when only rotational skills can be used.

ceq moveDown $((\mathrm{X}, \mathrm{Y}, \mathrm{Z})$, Skills 〈skill〉 Skill

〈type move 〈/type

〈subtype linear 〈/subtype

$\langle$ direction $\rangle$ vertical $\langle/$ direction $\rangle$ $\langle$ range $\rangle$ Range $\langle/$ range $\rangle$

$$
=\langle\text { skill }\rangle \text { Skill }
$$$$
\langle/ \text { skill }\rangle
$$

$\langle$ type move $\langle/$ type

〈subtype linear 〈/subtype

$\langle$ direction $\rangle$ vertical $\langle/$ direction $\rangle$

$\langle$ range $\rangle-$ float $($ deltaz $-\mathrm{Z})\langle/$ range $\rangle$

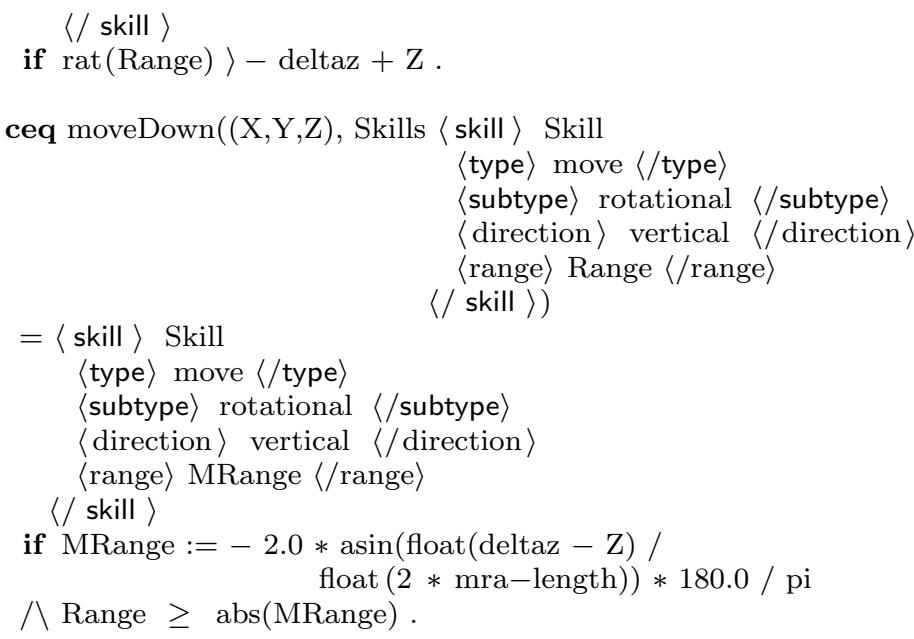

The moveDown takes as argument a three-dimensional position but only moves on the vertical axe, from the current position $\left(\delta_{z}\right)$ to the one specified $(\mathrm{Z})$. The equations first detect a skill which can be used for the movement (signified by the side condition of the equation) and then they create a new variant of the skill consti- 

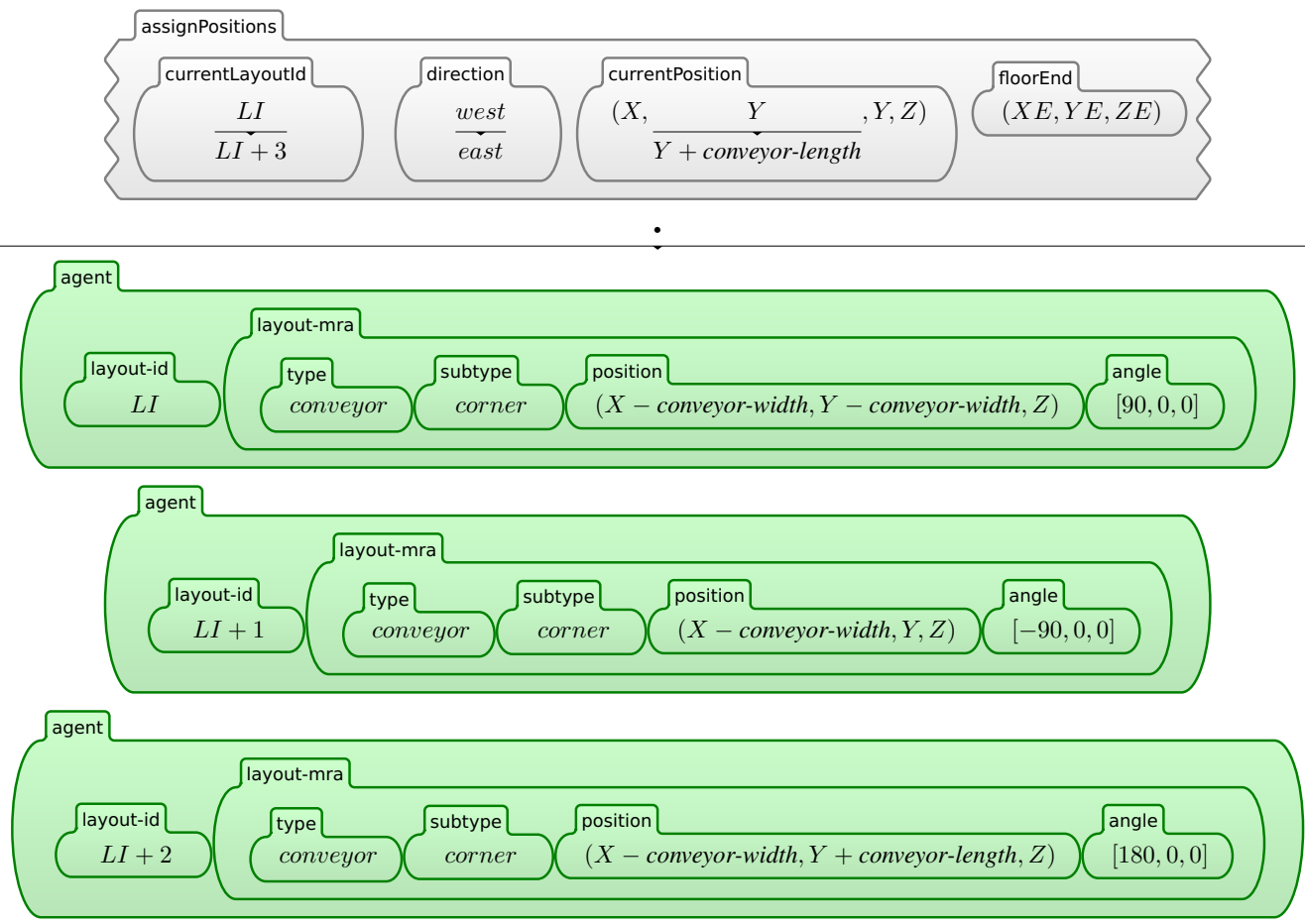

when $X$ - conveyor-length - conveyor-width $<0$ and $Y+$ conveyor-length + conveyor-width $\leq Y E$

Fig. 13 Switching the direction of the assembly line

tuting the actual operation, in which the range made available by the skill is replaced by the concrete range and direction required for the specific move operation. The equations defining the moveUp and moveFromTo operation follow a similar pattern.

The Maude implementation also includes definitions for the concrete conveyors movements, as well as for the humans loading/unloading the final product.

Without going into additional gory details here, the interface for deriving the LSAI is specified in Maude as follows:

$$
\begin{aligned}
& \text { eq deriveLSAI(SAgents, Cfg) } \\
& =\text { deriveLSAI(sortedList(SAgents), nil, Cfg, } \\
& \\
& \quad\langle\text { product }\rangle \text { nil }\langle/ \text { product }\rangle) .
\end{aligned}
$$

eq deriveLSAI(nil, Agents', Cfg, Product) $=\langle$ final-agents $\rangle$ Agents' $\langle/$ final-agents $\rangle$

$\langle$ final-product $\rangle$ Product $\langle/$ final-product $\rangle$.

The first equation defines the deriveLSAI function taking as arguments the set of agents obtained after the layout generation has completed and the remainder of the configuration, containing the specifications for parts, modules, and the assembly plan. This function delegates work to an auxiliary function (named deriveLSAI as well), which takes as arguments the list of agents sorted by task identifiers and the configuration, and uses the additional two arguments to hold the agents which have already been associated LSAIs to, as well as for dynamically constructing the final products. Once the processing has completed, making use of rules as those for the pick\&place operation mentioned above to associate LSAI information to all agents, then the second equation applies, presenting the resulting agents and the final product as a result.

\subsection{Example of an execution result}

For the case study presented in Section 3 and the modules listed in Section 5.1, Maude generates 47 possible solutions in just a few seconds. The final product generated by Maude, including the concrete positioning of all parts is presented in Appendix A.

Each solution also includes the coalition formed to solve each task, with their concrete positioning on the shop floor, and the conveyors linking them. The LSAI procedures are also represented in these agents, including the movements of the carrier and loading/unloading the product. The Maude generated output for the first solution describing these agents and their corresponding LSAI procedures is presented in Appendix B. Additionally, Figure 9 illustrates the procedure to assemble the product: 
It associates a person to load the carrier on the conveyor, satisfying task 1 . Then a conveyor carries the carrier eastward, where the coalition associated with task 1 picks the body-case from the feeder and places it on top of the carrier to complete task 2. The another conveyor carries the product eastward where the coalition associated with task 2 picks the tape-roll from the feeder and places it on top of the body case to complete task 3. Again, the product is carried eastward by another conveyor, where the coalition associated with task 3 picks the body-case from the feeder and places it on top of the tape roll to complete task 4 . Given that the eastward end of the shop floor is about to be reached, the direction of movement is changed first northward by using a corner conveyor, then a regular conveyor is used to move the product north to create enough separation space from the existing coalitions, and then again a corner conveyor is used to change the direction westward. A conveyor then transports the product westward, where a coalition associated with task 4 (and thus containing a gripper that is able to insert a screw) picks the screw and uses it to connect the existing parts on top of the carrier to complete task 5. Finally, the product is carried away westward by a conveyor at the end of which a person awaits to unload it.

As mentioned above, Maude generates 48 solutions for this GAP. The solutions differ from each other in that the available modules compose different coalitions, which then also reflects in the generated LSAI. For example, one difference between the first and the 25th solution is that in the 25 th solution, the coalition addressing task 2 contains robot $r 3$ instead of robot $r 1$, and thus the skills used for movements have to be rotational instead of linear; similarly, for task 3 , robot $r 1$ is used instead robot $r 2$, changing the movement type from rotational to linear; finally, for task 4, r2 is used instead of $r 3$, again changing the movement type to linear from rotational.

\section{Discussion and Conclusion}

The original formal specifications of Self-Organising Assembly Systems (SOAS) have been refined and improved; they are now richer, more detailed, and more realistic. Given a Generic Assembly Plan (GAP), a set of Manufacturing Resource Agents (MRA) as well as user preferences, the Maude software produces a self-organising shop-floor layout that is able to execute the required assembly operations, together with the Layout-Specific Assembly Instructions (LSAI).

The execution traces serve as a proof that Maude was able to find a solution, meaning that the Manufacturing Resource Agents (MRAs) can arrange themselves in a suitable way to execute the required assembly operation and thus to assemble the final product.

A valid question which the informed reader might ask is how to make sure that the obtained solutions are correct, and whether all possible executions are obtained. With respect to the first issue, we assume all of our rules, implemented as equations and rewrite rules in Maude, are faithfully modeling the rules and the intuition described in the paper. Moreover, given the constructive nature of the problem being addressed, any solution given by Maude can first be traced (to check that each rule applied correctly) and validated by effectively checking that the solution conforms to the problem. With respect to the technique being exhaustive, we do not see this as being crucial, as plenty of solutions are found nevertheless. The Maude rewrite engine does guarantee that all the behaviors of a rewrite specification are being explored using its search command if the rewrite system associated to it by orienting equations from left to right is confluent and terminating on its equational part, while its rules are coherent with regard to the equations (Clavel et al. 2007). Confluence of the equational part comes from the fact that the equational part is deterministic. This can be syntactically checked by noticing that equations are non-overlapping, i.e., no more than one left-hand-side matches the same part of a term to be rewritten concurrently. Termination is harder to prove, requiring decreasing orders; however, the specifications describe terminating processes, so intuitively rewriting by equations should converge. Moreover, if the equations were non-terminating, there are high chances that the rewrite process would be nonterminating too, and thus not able to produce any solution, which clearly is not the case. Finally, coherence itself is nontrivial to prove in general; however, it can be checked that the patterns used by our rewrite rules are normal forms with regard to the equations, which is a strong syntactic criterion for coherence.

While the current work is only preliminary, it could be transformed into a user-friendly software system that manufacturing companies could use to verify if their manufacturing resources are sufficient to assemble a product, and to generate one or many suggestions for how to arrange the shop-floor layout, as well as to produce a rough sketch of the necessary assembly movements of each robotic module. Refinements would then be made using the individual control software of each robot or existing system integration tools. An advantage from using the approach we suggest is that it considerably shortens and facilitates the assembly system design phase, both when setting up a new system (which may take up to six months) and when modifying an existing one. 
In the current version of the specification, the first step is to generate the layout for a given GAP, and then to derive the corresponding LSAI. This is suitable for a proof of concept, that such specifications and rules are capable of generating a layout. The currently implemented model is simplified and only searches for a viable solution, without giving any importance to performance characteristics or efficiency.

If the goal is to produce an optimised solution, the procedure could also be done the other way round: more specific assembly instructions could be specified or derived first, and then a suitable layout generated. The best results would be received iteratively: having a rough concept of a suitable layout, deriving suitable assembly instructions, then improving the layout, and adapting the instructions, and so forth.

The currently used sequence of movements for a 'pick\&place' operation is very simple; it is described in Section 5.2. In a more realistic implementations, more sophisticated sequences and algorithms could be used to produce movements that are optimised for each robot and its kinematic characteristics.

For the sake of simplicity, the system currently only generates a linear layout for a linear GAP; given a dependency graph for a more complicated assembly, potentially with several sub-assemblies to produce first, a suitable layout could be generated as well. Again, user preferences would guide what kind of layout would be designed.

The main accomplishment of this work is that an assembly system is able to design itself, that is, to select suitable modules, create coalitions to provide all composite skills needed to assemble the product, to arrange the coalitions in a shop floor layout, and to produce the robotic modules' movements (i.e. to derive the LSAI). Future work is directed towards mechanisms for self-management while the system is executing the assembly.

For this work to be useable in an industrial setting, it requires for the specifications to be translated into a multi-agent system that is able to communicate with the robots and the order processing system used by the manufacturing company. Suitable multiagent manufacturing shopfloor control systems exist, with CoBASA (Barata 2005) being the basis on which this work was developed. CoBASA has been augmented over the last few years, adding more autonomy at the module level and including features for a variety of self-* properties, including self-adaptivity, self-diagnosis and self-learning (Barata et al. 2010; Candido et al. 2012; Frei 2010; Ribeiro et al. 2008).

In terms of the formal specifications, future work includes the preparation of a template for the user to comfortably specify any type of desired layout as well as other preferences, and the specification of the selfmanagement process that governs the actual production execution, as opposed to the creation of the assembly system, which was covered in this article.

\section{References}

J.-P. Banâtre, P. Fradet, and D. Le Métayer. Gamma and the chemical reaction model: Fifteen years after. In $W M P$, volume 2235 of $L N C S$, pages 17-44. Springer, 2000.

J. Barata. Coalition based approach for shopfloor agility. Edições Orion, Amadora - Lisboa, 2005.

J. Barata, L. Ribeiro, and A.-W. Colombo. A service-oriented shop floor to support collaboration in manufacturing networks. In L. Benyoucef and B. Grabot, editors, Artificial Intelligence Techniques for Networked Manufacturing Enterprises Management, Springer Series in Advanced Manufacturing, pages 483-503. Springer London, 2010.

G. Berry and G. Boudol. The chemical abstract machine. Theoretical Computer Science, 96(1):217-248, 1998.

M. Boncheva, D.A. Bruzewicz, and G.M. Whitesides. Millimeter-scale self-assembly and its applications. Pure and Applied Chemistry, 75(5):621-630, 2003.

G. Candido, G. Di Orio, J. Barata, and S. Scholze. Adapter for self-learning production systems. In L. CamarinhaMatos, E. Shahamatnia, and G. Nunes, editors, Technological Innovation for Value Creation, volume 372 of IFIP Advances in Information and Communication Technology, pages 171-178. Springer Boston, 2012.

M. Clavel, M. Palomino, and A. Riesco. Introducing the ITP tool: a tutorial. Journal of Universal Computer Science, 12(11):1618-1650, 2006.

M. Clavel, F. Durán, S. Eker, P. Lincoln, N. Martí-Oliet, J. Meseguer, and C. Talcott. All About Maude - A HighPerformance Logical Framework: How to Specify, Program, and Verify Systems in Rewriting Logic. LNCS. Springer Verlag New York, Inc., Secaucus, NJ, USA, 2007.

G. Di Marzo Serugendo and R. Frei. Experience report in developing and applying a method for self-organisation to agile manufacturing. In IEEE Int. Conf. on Self-Adaptive and Self-Organizing Systems (SASO), Budapest, Hungary, 2010.

G. Di Marzo Serugendo and R. Frei. Self-awareness in agile assembly systems. Awareness magazine: self-awareness in autonomic systems. http: //www. awareness-mag. eu, March(12), 2012.

S. Eker, J. Meseguer, and A. Sridharanarayanan. The Maude LTL model checker and its implementation. In 10th Int. SPIN Workshop on Model Checking of Software, LNCS, pages 230-234. Springer, 2003.

A. Farzan, F. Chen, J. Meseguer, and G. Rosu. Formal analysis of Java programs in JavaFAN. In Computer Aided Verification (CAV), pages 501-505, 2004.

R. Frei. Self-organisation in Evolvable Assembly Systems. $\mathrm{PhD}$ thesis, Department of Electrical Engineering, Faculty of Science and Technology, Universidade Nova de Lisboa, Portugal, 2010.

R. Frei and G. Di Marzo Serugendo. Concepts in complexity engineering. Int. J. of Bio-Inspired Computation, 3(2): 123-139, 2011a. 
R. Frei and G. Di Marzo Serugendo. Advances in complexity engineering. Int. J. of Bio-Inspired Computation, 3(4): 199-212, 2011b.

R. Frei and G. Di Marzo Serugendo. Self-organising assembly systems. IEEE Transactions on Systems, Man and Cybernetics, Part C: Applications and Reviews, 41(6):885897, November 2011c.

R. Frei and G. Di Marzo Serugendo. The future of complexity engineering. To appear in Central European Journal of Engineering, 2012.

R. Frei, G. Di Marzo Serugendo, and J. Barata. Designing self-organization for evolvable assembly systems. In IEEE Int. Conf. on Self-Adaptive and Self-Organizing Systems (SASO), pages 97-106, Venice, Italy, 2008a.

R. Frei, B. Ferreira, and J. Barata. Dynamic coalitions for self-organizing manufacturing systems. In CIRP Int. Conf. on Intelligent Computation in Manufacturing Engineering (ICME), Naples, Italy, 2008b.

R. Frei, B. Ferreira, G. Di Marzo Serugendo, and J. Barata. An architecture for self-managing evolvable assembly systems. In IEEE Int. Conf. on Systems, Man, and Cybernetics (SMC), San Antonio, TX, USA, 2009.

R. Frei, G. Di Marzo Serugendo, and T.F. Serbanuta. Ambient intelligence in self-organising assembly systems using the chemical reaction model. J. of Ambient Intelligence and Humanized Computing, 1(3):163-184, 2010a.

R. Frei, N. Pereira, J. Belo, J. Barata, and G. Di Marzo Serugendo. Implementing self-organisation and selfmanagement in evolvable assembly systems. In IEEE Int. Symp. on Industrial Electronics (ISIE), pages 3527-3532, Bari, Italy, 2010b.

R. Frei, A. Tiwari, R. McWilliam, and A. Purvis. Self-healing technologies. IEEE Transactions on Systems, Man and Cybernetics, Part C: Applications and Reviews, 2012.

R. Gross and M. Dorigo. Self-assembly at the macroscopic scale. Proceedings of the IEEE, 96(9):1490-1508, sept. 2008.

ISTAG. Scenarios for ambient intelligence in 2010. information society technologies advisory group report. http: //www. cordis.lu/ist/istag.htm, 2001.

ISTAG. Ambient intelligence: from vision to reality. information society technologies advisory group report. http: //www. cordis.lu/ist/istag.htm, 2003.

J.O. Kephart and D.M. Chess. The vision of autonomic computing. IEEE Computer, 36(1):41-50, 2003.

J. Meseguer. Rewriting as a unified model of concurrency. In Concur Conf., volume 458 of $L N C S$, pages 384-400, Amsterdam, The Netherlands, 1990. Springer Berlin Heidelberg.

J. Meseguer. Conditional rewriting logic as a unified model of concurrency. Theoretical Computer Science, 96(1):73155, 1992.

M. Onori. Evolvable assembly systems - a new paradigm? In 33rd Int. Symposium on Robotics (ISR), pages 617-621, Stockholm, Sweden, 2002.

M. Onori, D. Semere, and J. Barata. Evolvable assembly systems: From evaluation to application. In A. Azvedo, editor, Innovation in Manufacturing Networks, volume 266 of IFIP International Federation for Information Processing, pages 205-214. Springer, 2008.

M. Onori, D. Semere, and B. Lindberg. Evolvable systems: an approach to self-X production. Int. Journal of Computer Integrated Manufacturing, 24(5):506-516, 2011.

D. Phili and J.F. Stoddart. Self-assembly in natural and unnatural systems. Applied Chemistry: International Edition, 35(11):1154-1196, 1996.
L. Ribeiro, J. Barata, and A. Colombo. MAS and SOA: A case study exploring principles and technologies to support self-properties in assembly systems. In $2 n d I E E E$ Int. Conf. on Self-Adaptive and Self-Organizing Systems Workshops (SASOW), pages 192-197, oct. 2008.

R. Sasse and J. Meseguer. Java+ITP: A verification tool based on Hoare logic and algebraic semantics. In G. Denker and C.L. Talcott, editors, 6th Int. Workshop on Rewriting Logic and its Applications (WRLA), volume 176(4) of Electronic Notes in Theoretical Computer Science, pages 29-46, 2007.

Traian Florin Șerbănuță, Grigore Roșu, and José Meseguer. A rewriting logic approach to operational semantics. Information and Computation, 207(2):305-340, 2009.

M. Ulieru and R. Doursat. Emergent engineering: A radical paradigm shift. J. of Autonomous and Adaptive Communications Systems, 4(1):39-60, 2011. 
A The final product as generated by the Maude specification

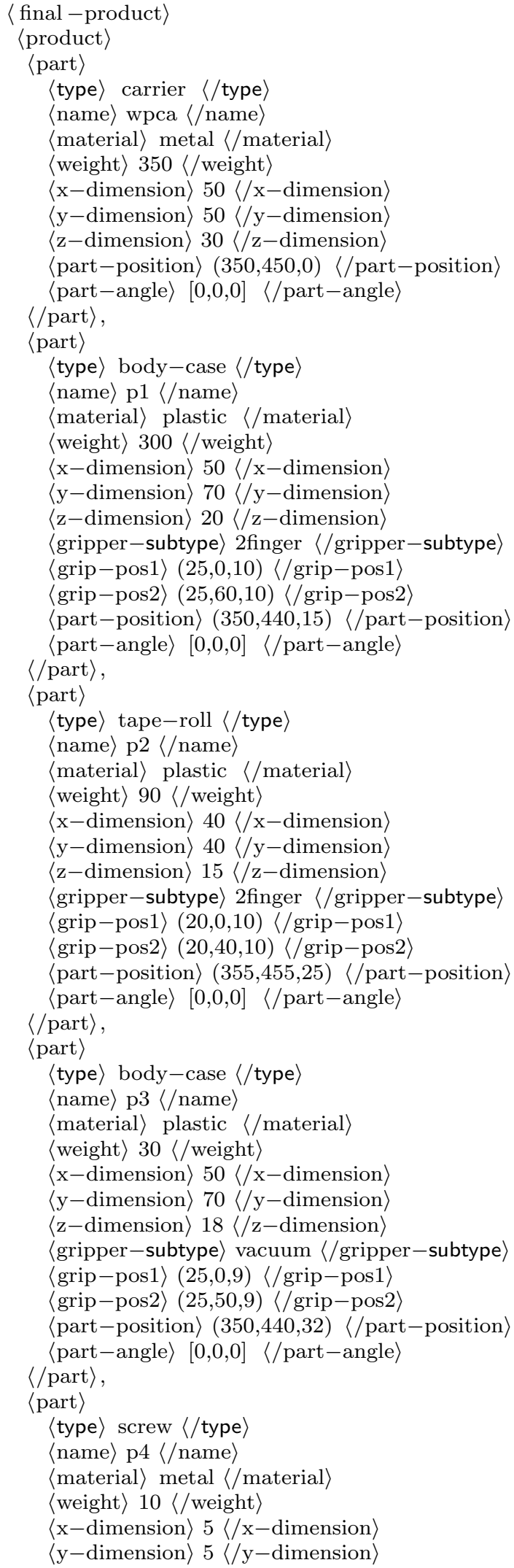

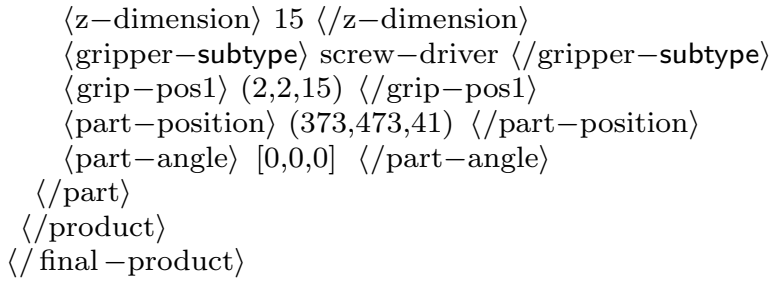

B A solution for the generation of the LSAI as being output by Maude

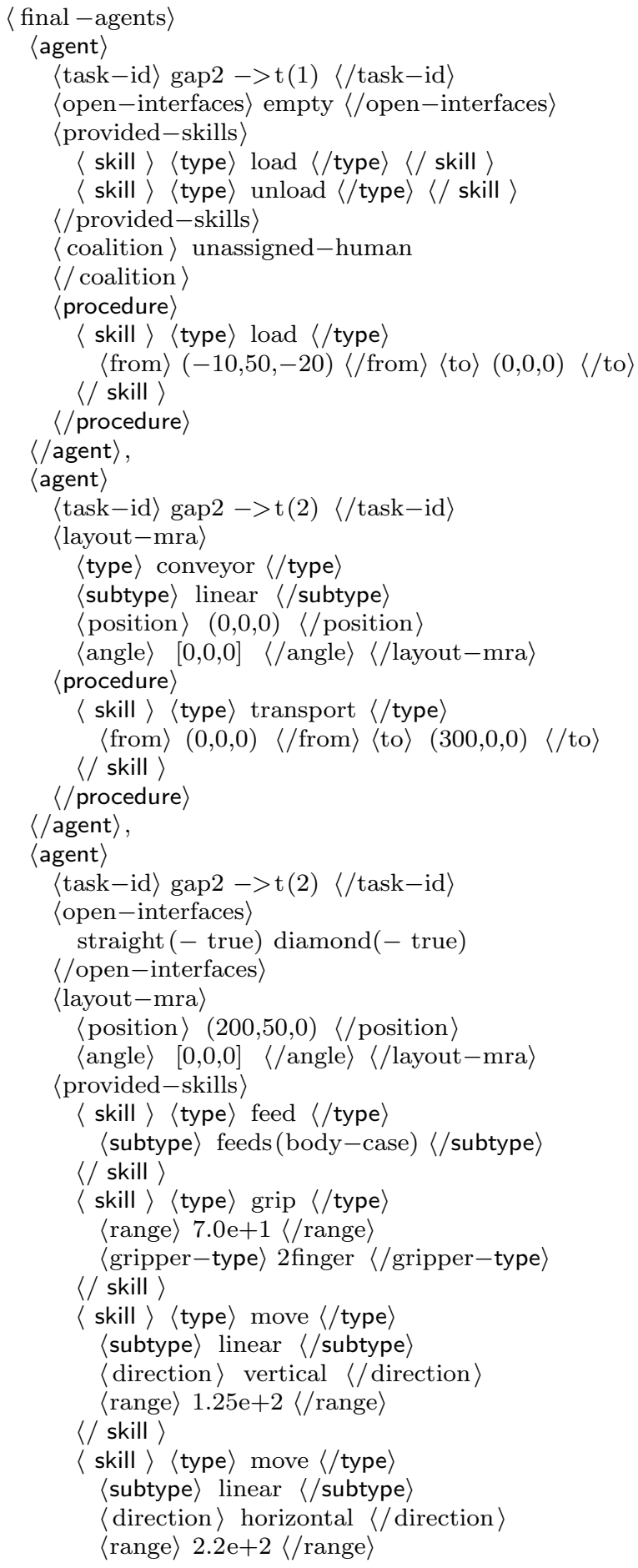




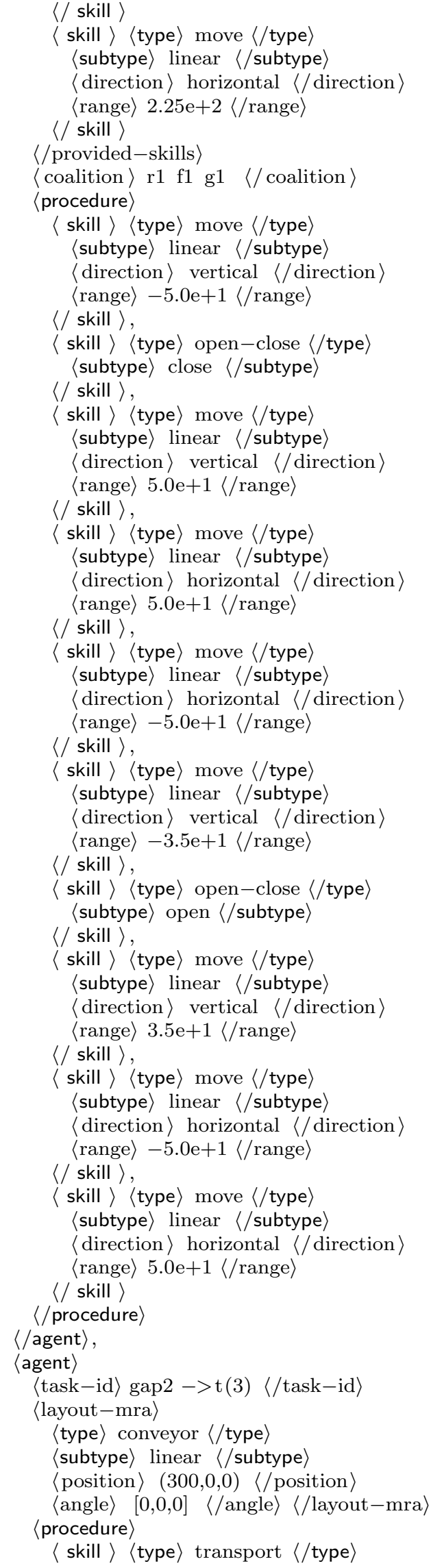

$\langle$ from $\rangle(300,0,0)\langle/$ from $\rangle\langle$ to $\rangle(600,0,0)\langle/$ to $\rangle$ $\langle/$ skill $\rangle$

$\langle/$ procedure $\rangle$

$\langle/$ agent $\rangle$,

〈agent

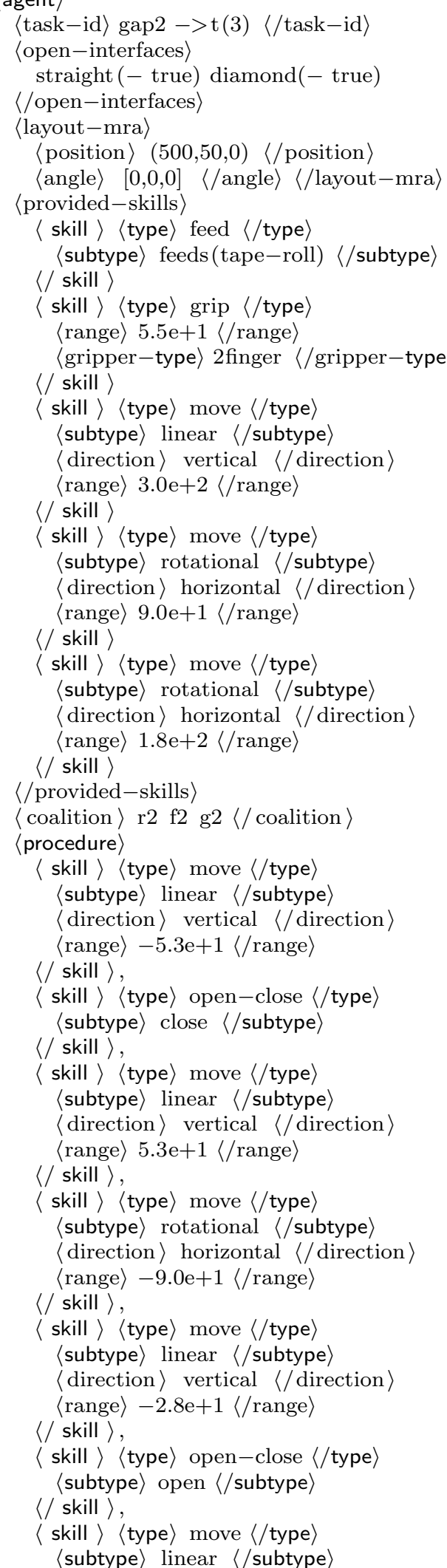




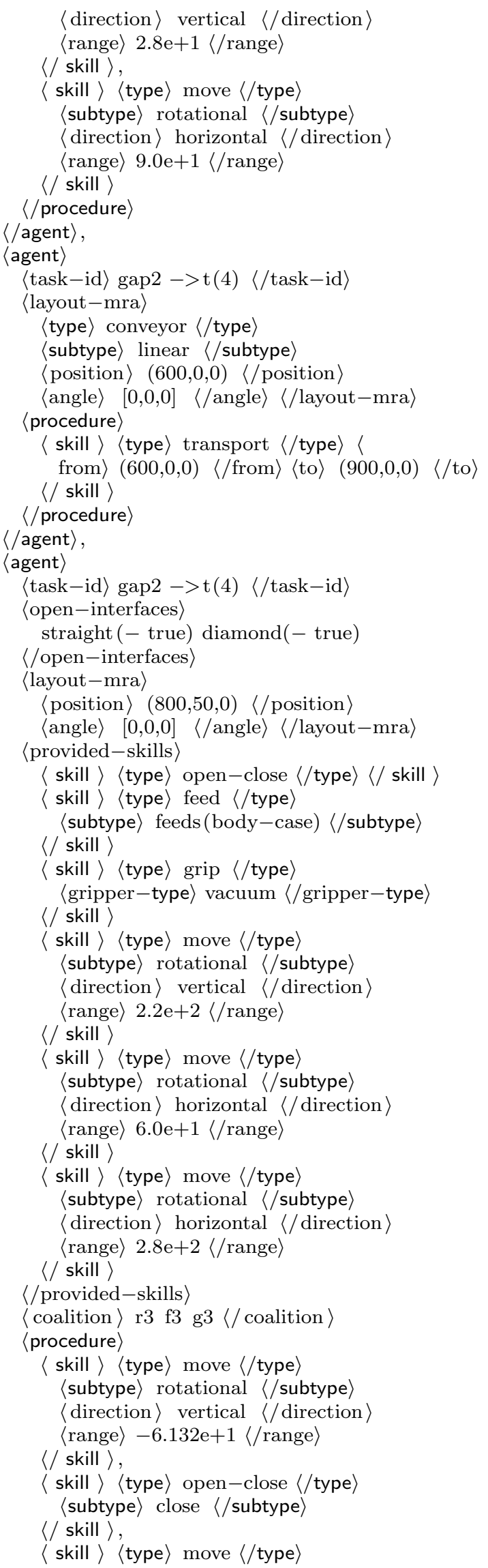

〈subtype rotational 〈/subtype

$\langle$ direction $\rangle$ vertical $\langle/$ direction $\rangle$

$\langle$ range $\rangle 6.132 \mathrm{e}+1\langle/$ range $\rangle$

$\langle/$ skill $\rangle$,

$\langle$ skill 〉 〈type move $\langle/$ type

〈subtype rotational 〈/subtype

$\langle$ direction〉 horizontal $\langle/$ direction $\rangle$

$\langle$ range $\rangle-9.0 \mathrm{e}+1\langle/$ range $\rangle$

$\langle/$ skill $\rangle$,

$\langle$ skill $\rangle\langle$ type $\rangle$ move $\langle/$ type

$\langle$ subtype $\rangle$ rotational $\langle/$ subtype $\rangle$

$\langle$ direction $\rangle$ vertical $\langle/$ direction $\rangle$

$\langle$ range $\rangle-2.19 \mathrm{e}+1\langle/$ range $\rangle$

$\langle/$ skill $\rangle$,

$\langle$ skill $\rangle\langle$ type $\rangle$ open-close $\langle/$ type

〈subtype open 〈/subtype

$\langle/$ skill $\rangle$,

$\langle$ skill $\rangle\langle$ type $\rangle$ move $\langle/$ type

$\langle$ subtype $\rangle$ rotational $\langle/$ subtype $\rangle$

$\langle$ direction $\rangle$ vertical $\langle/$ direction $\rangle$

$\langle$ range $\rangle 2.19 \mathrm{e}+1\langle/$ range $\rangle$

$\langle/$ skill $\rangle$.

$\langle$ skill $\rangle\langle$ type $\rangle$ move $\langle/$ type

〈subtype rotational 〈/subtype

$\langle$ direction〉 horizontal $\langle/$ direction

$\langle$ range $\rangle 9.0 \mathrm{e}+1\langle/$ range $\rangle$

$\langle/$ skill $\rangle$

$\langle/$ procedure $\rangle$

$\langle/$ agent $\rangle$,

〈agent $\rangle$

$\langle$ task-id $\rangle$ gap2 $->$ t $(17 / 4)\langle/$ task-id $\rangle$

$\langle$ layout-mra

$\langle$ type conveyor $\langle/$ type

〈subtype corner 〈/subtype

$\langle$ position〉 $(900,0,0)$ 〈/position

$\langle$ angle $\quad[0,0,0] \quad\langle/$ angle $\rangle\langle/$ layout-mra $\rangle$

$\langle$ procedure $\rangle$

$\langle$ skill $\rangle\langle$ type $\rangle$ transport $\langle/$ type

$\langle$ from $\rangle(900,0,0)\langle/$ from $\rangle\langle$ to $\rangle(950,0,0)\langle/$ to $\rangle$

$\langle/$ skill $\rangle$

$\langle/$ procedure $\rangle$

$\langle/$ agent $\rangle$,

〈agent $\rangle$

$\langle$ task-id $\rangle$ gap2 $->$ t $(9 / 2)\langle/$ task-id $\rangle$

$\langle$ layout-mra

$\langle$ type conveyor $\langle/$ type

〈subtype〉 linear 〈/subtype

$\langle$ position $\rangle(900,50,0)\langle/$ position $\rangle$

$\langle$ angle $\rangle[-90,0,0]\langle/$ angle $\rangle\langle/$ layout - mra $\rangle$

$\langle$ procedure $\rangle$

〈skill 〉 〈type transport 〈/type

$\langle$ from $\rangle(950,0,0)\langle/$ from $\rangle\langle$ to $\rangle(950,300,0)\langle/$ to $\rangle$

$\langle/$ skill $\rangle$

$\langle/$ procedure $\rangle$

$\langle/$ agent $\rangle$,

〈agent $\rangle$

$\langle$ task-id $\rangle$ gap2 $->$ t $(19 / 4)\langle/$ task-id $\rangle$

$\langle$ layout-mra

$\langle$ type $\rangle$ conveyor $\langle/$ type $\rangle$

〈subtype corner $\langle/$ subtype

〈position $\rangle(900,350,0)$ 〈/position $\rangle$

$\langle$ angle $[-90,0,0]\langle/$ angle $\rangle\langle/$ layout-mra

$\langle$ procedure

$\langle$ skill $\rangle\langle$ type $\rangle$ transport $\langle/$ type

$\langle$ from $\rangle(950,300,0)\langle/$ from $\rangle\langle$ to $\rangle(950,350,0)\langle/$ to $\rangle$

$\langle/$ skill $\rangle$ 


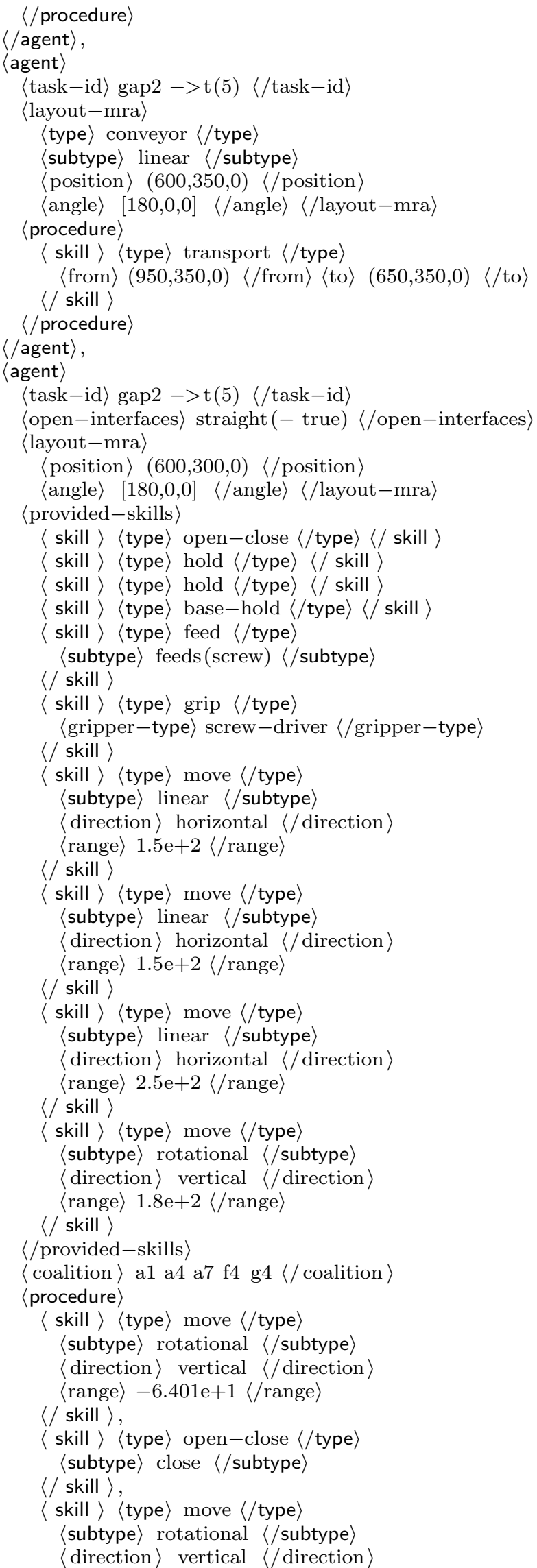

$\langle$ range $\rangle 6.401 \mathrm{e}+1\langle/$ range $\rangle$

$\langle/$ skill $\rangle$,

$\langle$ skill $\rangle\langle$ type $\rangle$ move $\langle/$ type

〈subtype linear 〈/subtype

$\langle$ direction〉 horizontal $\langle/$ direction $\rangle$

$\langle$ range $\rangle-5.0 \mathrm{e}+1\langle/$ range $\rangle$

$\langle/$ skill $\rangle$,

〈 skill 〉〈type move 〈/type

〈subtype linear 〈/subtype

$\langle$ direction $\rangle$ horizontal $\langle/$ direction $\rangle$

$\langle$ range $\rangle 5.0 \mathrm{e}+1\langle/$ range $\rangle$

$\langle/$ skill $\rangle$,

$\langle$ skill $\rangle\langle$ type $\rangle$ move $\langle/$ type $\rangle$

〈subtype $\rangle$ rotational $\langle/$ subtype

$\langle$ direction $\rangle$ vertical $\langle/$ direction $\rangle$

$\langle$ range $\rangle-1.3786 \mathrm{e}+1\langle/$ range $\rangle$

$\langle/$ skill $\rangle$,

$\langle$ skill $\rangle\langle$ type $\rangle$ open-close $\langle/$ type

$\langle$ subtype $\rangle$ open $\langle/$ subtype $\rangle$

$\langle/$ skill $\rangle$,

$\langle$ skill $\rangle\langle$ type $\rangle$ move $\langle/$ type

〈subtype rotational 〈/subtype

$\langle$ direction $\rangle$ vertical 〈/direction〉

$\langle$ range $\rangle 1.378 \mathrm{e}+1\langle/$ range $\rangle$

$\langle/$ skill $\rangle$,

$\langle$ skill $\rangle\langle$ type $\rangle$ move $\langle/$ type $\rangle$

〈subtype linear 〈/subtype

$\langle$ direction〉 horizontal $\langle/$ direction $\rangle$

$\langle$ range $\rangle$.0e+1 $\langle/$ range $\rangle$

$\langle/$ skill $\rangle$

$\langle$ skill $\rangle\langle$ type move $\langle/$ type

〈subtype linear 〈/subtype

〈direction〉 horizontal 〈/direction〉

$\langle$ range $\rangle-5.0 \mathrm{e}+1\langle/$ range $\rangle$

$\langle/$ skill $\rangle$

$\langle/$ procedure

$\langle/$ agent $\rangle$,

〈agent>

$\langle$ task-id $\rangle$ gap2 $->$ t $(6)\langle/$ task-id $\rangle$

$\langle$ layout-mra $\rangle$

$\langle$ type conveyor $\langle/$ type $\rangle$

〈subtype linear 〈/subtype)

〈position〉 $(300,350,0)$ 〈 position〉

$\langle$ angle $\rangle[180,0,0]\langle/$ angle $\rangle\langle/$ layout-mra $\rangle$

$\langle$ procedure $\rangle$

$\langle$ skill $\rangle\langle$ type $\rangle$ transport $\langle/$ type $\rangle$

$\langle$ from $\rangle(650,350,0)\langle/$ from $\rangle\langle$ to $\rangle(350,350,0)\langle/$ to $\rangle$

$\langle/$ skill $\rangle$

$\langle/$ procedure $\rangle$

$\langle/$ agent $\rangle$,

$\langle$ agent $\rangle$

$\langle$ task-id $\rangle$ gap2 $->\mathrm{t}(6)\langle/$ task-id $\rangle$

$\langle$ open-interfaces $\rangle$ empty $\langle/$ open-interfaces $\rangle$

$\langle$ layout-mra

$\langle$ position〉 $(300,300,0)$ 〈/position〉

$\langle$ angle $\rangle[180,0,0] \quad\langle/$ angle $\rangle$ /layout-mra

$\langle$ provided-skills $\rangle$

$\langle$ skill $\rangle\langle$ type $\rangle$ load $\langle/$ type $\rangle\langle/$ skill $\rangle$

$\langle$ skill $\rangle\langle$ type $\rangle$ unload $\langle/$ type $\rangle\langle/$ skill $\rangle\langle/$ provided-skills $\rangle$

$\langle$ coalition $\rangle$ unassigned-human

$\langle/$ coalition $\rangle$

$\langle$ procedure $\rangle$

$\langle$ skill $\rangle\langle$ type $\rangle$ unload $\langle/$ type

$\langle$ from $\rangle(350,350,0)\langle/$ from $\rangle\langle$ to $\rangle(350,450,0)\langle/$ to $\rangle$

$\langle/$ skill $\rangle$

$\langle/$ procedure 
〈/agent

$\langle/$ final-agents $\rangle$ 